\title{
On the applicability of electrical impedance indices to characterize the condition of the oral mucosa
}

\author{
I. Lacković, Z. Stare \& T. Protulipac \\ Faculty of Electrical Engineering and Computing, \\ University of Zagreb, Croatia
}

\begin{abstract}
In recent years efforts have been put on the development of objective, noninvasive and inexpensive method to evaluate the condition of the oral mucosa. It was demonstrated in some special cases (experimental conditions, induced irritation reactions) that electrical impedance techniques have the potential of fulfilling these requirements. The aim of our study was to compare the information contained in baseline impedance values with the information extracted by impedance indices proposed by Ollmar et al. [10], and to evaluate these indices as a data reduction technique. Therefore, we measured impedance magnitude and phase in the range of $30 \mathrm{~Hz}$ to $1 \mathrm{MHz}$ at ten different points of the oral mucosa. Measurements were performed on a group of 20 subjects. From impedance data at $20 \mathrm{kHz}$ and $500 \mathrm{kHz}$ we calculated impedance indices $M I X$, $P I X, R I X$ and $I M I X$, and performed statistical analysis. We have demonstrated mathematically that $M I X, R I X$ and $I M I X$ form a redundant set of indices i.e. any of them can be calculated from other two. This disagrees with the essence of data reduction. Experimental results have shown that indices $R I X$ and $I M I X$ behave as downscaled index $M I X$, which indeed is the most valuable, intuitively well defined, and from the instrumentation point of view easiest to measure. We have also found that, due to its extremely high variability, index $P I X$ has no practical value (except maybe in cases when direct reference from the same subject is available). The main conclusion of our study is that the proposed set of indices is not fully satisfactory nor could be generally accepted as data reduction technique. Their applicability for characterization of the mucosal tissue is limited. The probe being used for impedance measurement plays an important role and indices should be defined anew for every probe design.
\end{abstract}




\section{Introduction}

Assessment of the condition of the oral mucosa is routinely performed by visual inspection. This evaluation is qualitative, undoubtedly subjective and limited to visible alterations of the superficial mucosal tissue. However, there are some pathophysiological conditions of the oral cavity characterized by normally appearing mucosa. Mouth dryness, burning mouth syndrome etc. are just some examples. Therefore, there is a need for noninvasive, objective and easy to use method that could help to evaluate both visible and invisible reactions of the oral mucosa.

Bioelectrical impedance techniques have been successfully used for tissue characterization for years [1,2]. Some investigators have proposed that impedance methods could be also employed for diagnostic or screening purposes and various applications have been reported (i.e. characterization of breast cancer tissues, assessment of muscle ischemia level, etc.) [3].

Impedance measurements in the oral cavity were not performed until recently. The first contributions came from Nilsson et al. [4], Ollmar et al. [5] and Nicander et al. [6,7]. Results of their research demonstrated that method based on electrical impedance measurements is capable of detecting irritation reactions of the oral mucosa below the visible threshold. All these studies were performed in healthy young volunteers and the irritation reactions were induced by exposure of the oral mucosa to different irritants. To extract information from impedance spectra they used impedance indices initially introduced in a similar study on induced irritant skin reactions [8,9]. In a review on information extraction from impedance spectra of biological tissue, Ollmar claimed that these four indices extract most of the information in the relevant part of the complex impedance spectrum of skin and oral mucosa [10]. Recently we presented our preliminary results of electrical impedance measurements at various regions of normal oral mucosa in the frequency range of $30 \mathrm{~Hz}$ to $1 \mathrm{MHz}$ [11]. To the best of our knowledge, that was the first contribution that came from authors outside the Swedish group. In this study our aim was to analyse the impedance indices as data reduction tool, to compare the information contained in baseline impedance values with the information extracted by impedance indices, and to determine reference values of impedance indices that would be used when data from reference sites on the same subject are not available.

\section{Materials and Methods}

\subsection{Experimental protocol}

\subsubsection{Subjects}

A group of 20 healthy subjects, 11 males and 9 females, participated in this study. Ages ranged from 22 to 74 years (median: 53 yrs., lower quartile: 43 yrs., upper quartile: 68 yrs.). Prior to the measurement every subject had undergone a detailed examination to exclude the presence of oral lesions. Measurements were performed in the dentist's office in a quite atmosphere. The subjects were 
recruited among the patients during their regular check-up visits. Every participant gave a written consent before the measurement.

\subsubsection{Instrumentation and data acquisition}

Precision LCR meter HP 4284A (Hewlett-Packard), supplied with our specially designed intraoral probe [11] was used for impedance measurement. The impedance magnitude and phase were measured in the frequency range between $30 \mathrm{~Hz}$ and $1 \mathrm{MHz}$ at ten approximately logarithmically spaced frequencies. Before the measurement corrections were made to compensate for the parasitic capacitance of the probe and the connecting wires. Impedance meter was connected to a portable computer via HPIB interface. A program was developed in HP-VEE 4.0 environment that enabled fully automated measurement, immediate display of impedance spectra on computer display and data storage to files for further analysis. Measurement and data acquisition for the whole frequency range took approximately $10 \mathrm{~s}$ for each oral region. Consequently data collection from ten explored regions was completed within 3-4 min.

\subsubsection{Explored regions of the oral mucosa}

Impedance measurements were made at ten different regions of the oral mucosa. Explored regions, designated with squares in Figure 1, included: lower lip
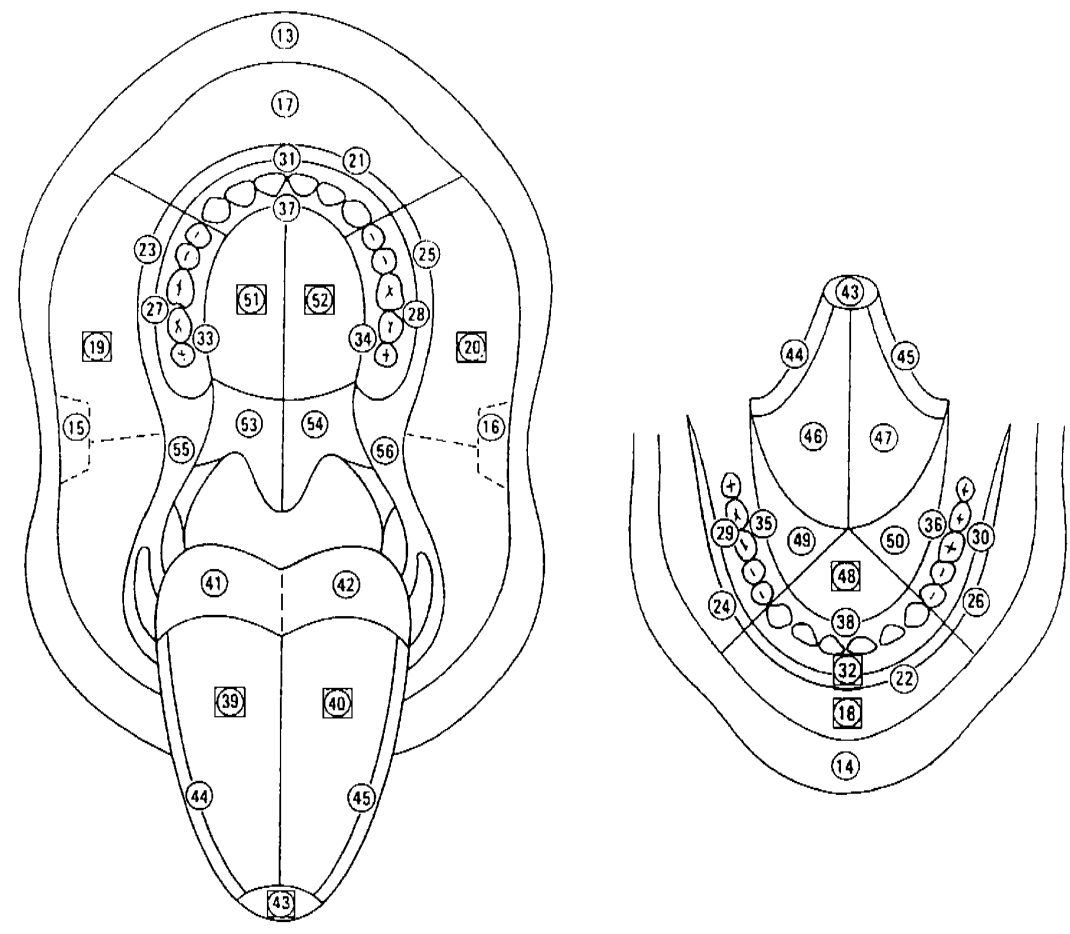

Figure 1: Regions of the oral mucosa (according to Roed-Petersen and Renstrup [12]). Squares indicate the locations of the measuring points. 


\section{4}

mucosa (R-18), right buccal mucosa (R-19), left buccal mucosa (R-20), vestibular mandibular gingiva (R-32), right dorsal side of the tongue (R-39), left dorsal side of the tongue (R-40), the tip of the tongue (R-43), floor of the mouth (R-48), right side of the hard palate (R-51) and left side of the hard palate (R-52). Topographical classification of the oral mucosa used here has been introduced by Roed-Petersen and Restrup, and is accepted by the World Health Organization [12].

\subsection{Impedance indices}

\subsubsection{Definition}

In order to perform data reduction and to extract relevant information from the impedance spectrum Ollmar and his co-workers have proposed four impedance indices: the magnitude index $(M I X)$, the phase index $(P I X)$, the real part index $(R I X)$ and the imaginary part index $(I M I X)$ [10]. The indices are defined as follows:

$$
\begin{aligned}
& M I X=\frac{|Z|_{20 \mathrm{kHz}}}{|Z|_{500 \mathrm{kHz}}} \\
& P I X=\varphi_{20 \mathrm{kHz}}-\varphi_{500 \mathrm{kHz}} \\
& R I X=\frac{\operatorname{Re} Z_{20 \mathrm{kHz}}}{|Z|_{500 \mathrm{kHz}}} \\
& I M I X=\frac{\operatorname{Im} Z_{20 \mathrm{kHz}}}{|Z|_{500 \mathrm{kHz}}}
\end{aligned}
$$

where $|Z|$ is the impedance magnitude, $\varphi$ is the impedance phase (in degrees), $\operatorname{Re} Z$ is the real and $\operatorname{Im} Z$ is the imaginary part of the electric impedance, at the frequencies indicated in the subscripts.

\subsubsection{Theoretical considerations}

Index $M I X$ can be interpreted as dimensionless quantity proportional to the slope of magnitude spectrum between $20 \mathrm{kHz}$ and $500 \mathrm{kHz}$. Index $P I X$ measures change of phase between the same frequencies. Indices $R I X$ and $I M I X$ contain basically the same information as real and imaginary part of impedance at $20 \mathrm{kHz}$ respectively, but expressed dimensionless due to scaling by $|Z|_{500 \mathrm{kHz}}$.

To gain more insight into the indices, their meaning and relations, we performed some simple calculations.

Since

$$
\operatorname{Re} Z=|Z| \cos \varphi \quad \text { and } \quad \operatorname{Im} Z=|Z| \sin \varphi
$$

substitution of eqn (5a) to eqn (3) and eqn (5b) to eqn (4) yield:

$$
R I X=\frac{|Z|_{20 \mathrm{kHz}}}{|Z|_{500 \mathrm{kHz}}} \cos \varphi_{20 \mathrm{kHz}} \quad I M I X=\frac{|Z|_{20 \mathrm{kHz}}}{|Z|_{500 \mathrm{kHz}}} \sin \varphi_{20 \mathrm{kHz}} .
$$


Taking into account the definition of $M I X$ given by eqn (1), eqns (6a,b) can now be written as follows:

$$
R I X=M I X \cos \varphi_{20 \mathrm{kHz}} \quad I M I X=M I X \sin \varphi_{20 \mathrm{kHz}} .
$$

These two equations show that $R I X$ and $I M I X$ depend on $M I X$ and $\varphi_{20 \mathrm{kHz}}$. Also, since measured impedance of biological tissues is always capacitive, $R I X$ will be always positive and $I M I X$ always negative.

By squaring eqns (7a) and ( $7 b$ ), and then by adding them we come to a simple formula:

$$
R I X^{2}+I M I X^{2}=M I X^{2}
$$

that relates all three indices and proves that $R I X, I M I X$ and $M I X$ form a redundant (overcomplete) set, what means that any of these indices can be calculated from other two.

In our opinion, the essence of data reduction should be to obtain the representation with minimal number of independent components.

\subsubsection{Calculation of impedance indices}

Measured impedance data did not allow for the direct calculation of impedance indices since our measuring frequencies did not include $20 \mathrm{kHz}$ and $500 \mathrm{kHz}$. Therefore we interpolated magnitude and phase data from the nearest neighbouring values i.e. magnitude and phase at $20 \mathrm{kHz}$ were interpolated from measured values at $10 \mathrm{kHz}$ and $30 \mathrm{kHz}$, while magnitude and phase at $500 \mathrm{kHz}$ were interpolated from measured values at $300 \mathrm{kHz}$ and $1 \mathrm{MHz}$. For impedance magnitude data $\log$-log interpolation was used, whereas for phase data log-lin interpolation was used. In our case, these interpolations were much more appropriate than simple linear interpolation.

\subsection{Statistical analysis}

Both male and female subjects were treated together as a single experimental group. For all measuring frequencies and all explored oral regions descriptive statistical measures of impedance magnitude and phase were calculated. The same calculations were carried out for impedance indices.

In order to assess the variability of impedance indices the coefficient of variation was used. The coefficient of variation is a measure of relative dispersion and is given by:

$$
\text { Coefficient of variation }(\%)=\frac{\text { standard deviation }}{\text { mean }} \times 100 \text {. }
$$

The usage of the coefficient of variation lies in the fact that the mean and standard deviation tend to change together in many experiments.

Comparison of various regions was performed using the one-way ANOVA followed by post-hoc multiple comparisons (Tukey's HSD test and GamesHowell test when equal variances are not assumed). Shapiro-Wilk's test was used to test the normality of distributions. 


\section{Results and discussion}

\subsection{Impedance magnitude and phase spectra}

Calculated mean values and corresponding 95\% confidence intervals of impedance magnitude and phase for all frequencies and all explored regions are shown in Figure 2. Shaded rectangles, which represent the region between $20 \mathrm{kHz}$ and $500 \mathrm{kHz}$, clearly point how limited frequency range is covered by the impedance indices and how different impedance behaviour is found outside that frequency range. Impedance magnitude is highest at the hard palate (R-51, $\mathrm{R}-52$ ), lowest at the tongue dorsum and tip (R-39, R-40, R-43), and somewhere between these two at all other regions including buccal mucosa (R-19, R-20), floor of the mouth (R-48), lower lip mucosa (R-18) and vestibular mandibular gingiva (R-32) which all have very similar impedance. These differences are more prominent between $1 \mathrm{kHz}$ and $10 \mathrm{kHz}$, and outside that range tend to decrease. Above $100 \mathrm{kHz}$ magnitude spectra do not exhibit differences between hard palate and other regions except the tongue. Phase spectra show similar results, but in different frequency range and not so prominently.

Such impedance behaviour is the consequence of different tissue structure found in the oral cavity (keratinised, non-keratinised, multi-layered epithelium). As expected, no statistically significant difference was found between impedance magnitudes at symmetrically opposite locations (i.e. between R-51 and R-52, between R-39 and R-40, and between R-19 and R-20), while significant difference exists in a broad frequency range between tongue, hard palate and other regions. Differences between buccal mucosa, floor of the mouth, lower lip mucosa and vestibular mandibular gingiva were insignificant.

\subsection{Impedance indices}

Impedance indices, presented as box-whisker plots for various oral regions, are shown in Figure 3. Since $I M I X$ is always negative, we neglect the sign and treat $I M I X$ as its absolute value.

The plots reveal that $R I X$ and $I M I X$ resemble very much to $M I X$ and all of them contain basically the same information. Indices $P I X$ exhibit strange behaviour that does not correspond to differences between oral regions that were found in impedance spectra. Our results for $M I X$ and $I M I X$ are in good qualitative agreement with the results reported by Nicander et al. [6], although the values are slightly different. Great discrepancy exists for $P I X$ at all oral regions and for $R I X$ at the hard palate and at the floor of the mouth.

Presentation of all statistical results would take too much space, so we give a brief summary. The same findings as for impedance spectra (significant difference between hard palate, tongue and other regions) were found only for $M I X$ and $R I X$, and in part for $I M I X$. Index $P I X$ showed for example no difference between hard palate and tongue, which definitely exists in impedance data, and at the same time showed significant difference between vestibular mandibular gingiva and floor of the mouth, which was insignificant in impedance data. 

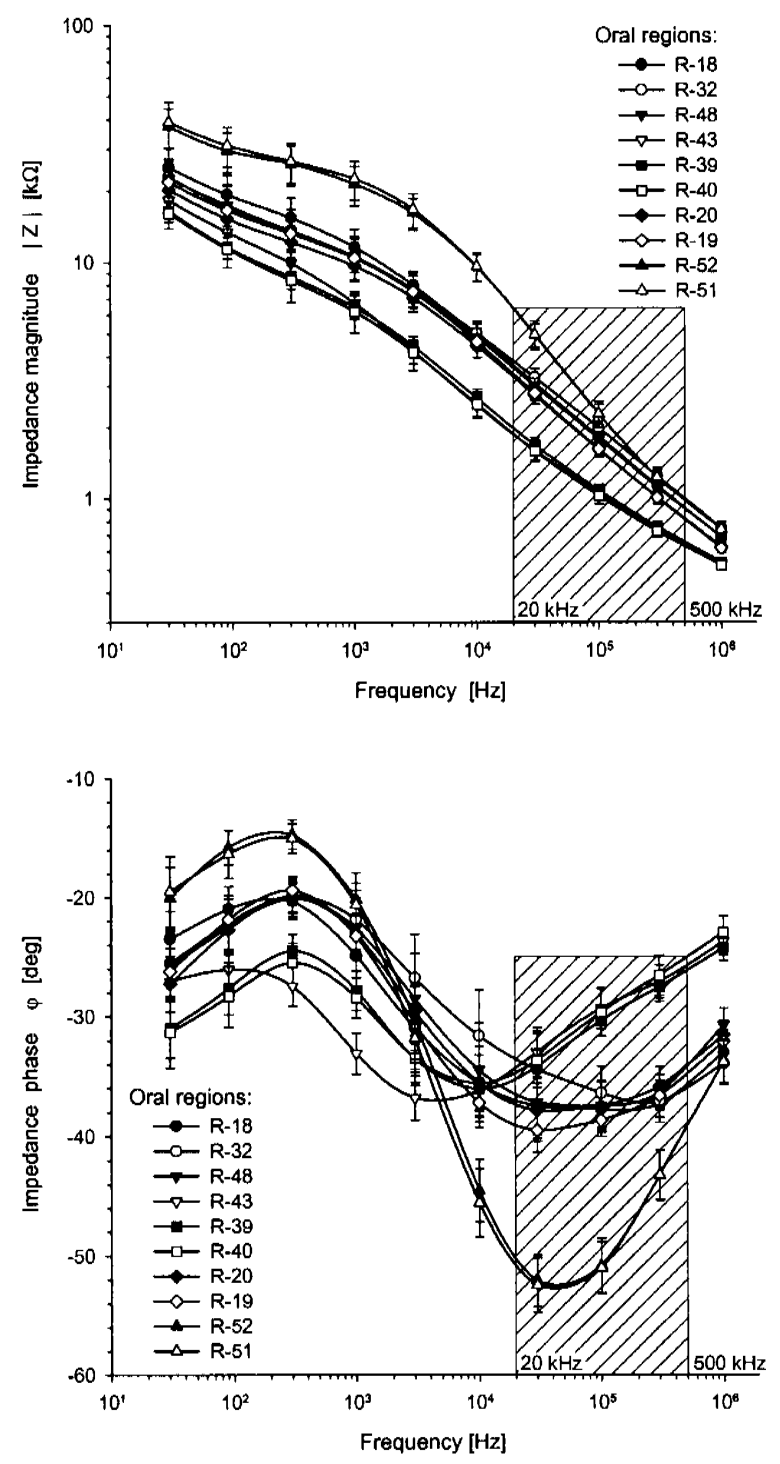

Figure 2: Impedance magnitude $|Z|$ and phase $\varphi$ spectra at various regions of the oral mucosa. Shaded rectangles represent frequency range between $20 \mathrm{kHz}$ and $500 \mathrm{kHz}$. Impedance indices $M I X, P I X$ RIX and $I M I X$ are calculated from impedance data at these two frequencies. Symbols represent mean and error bars the $95 \%$ confidence interval of the mean for our experimental group. 

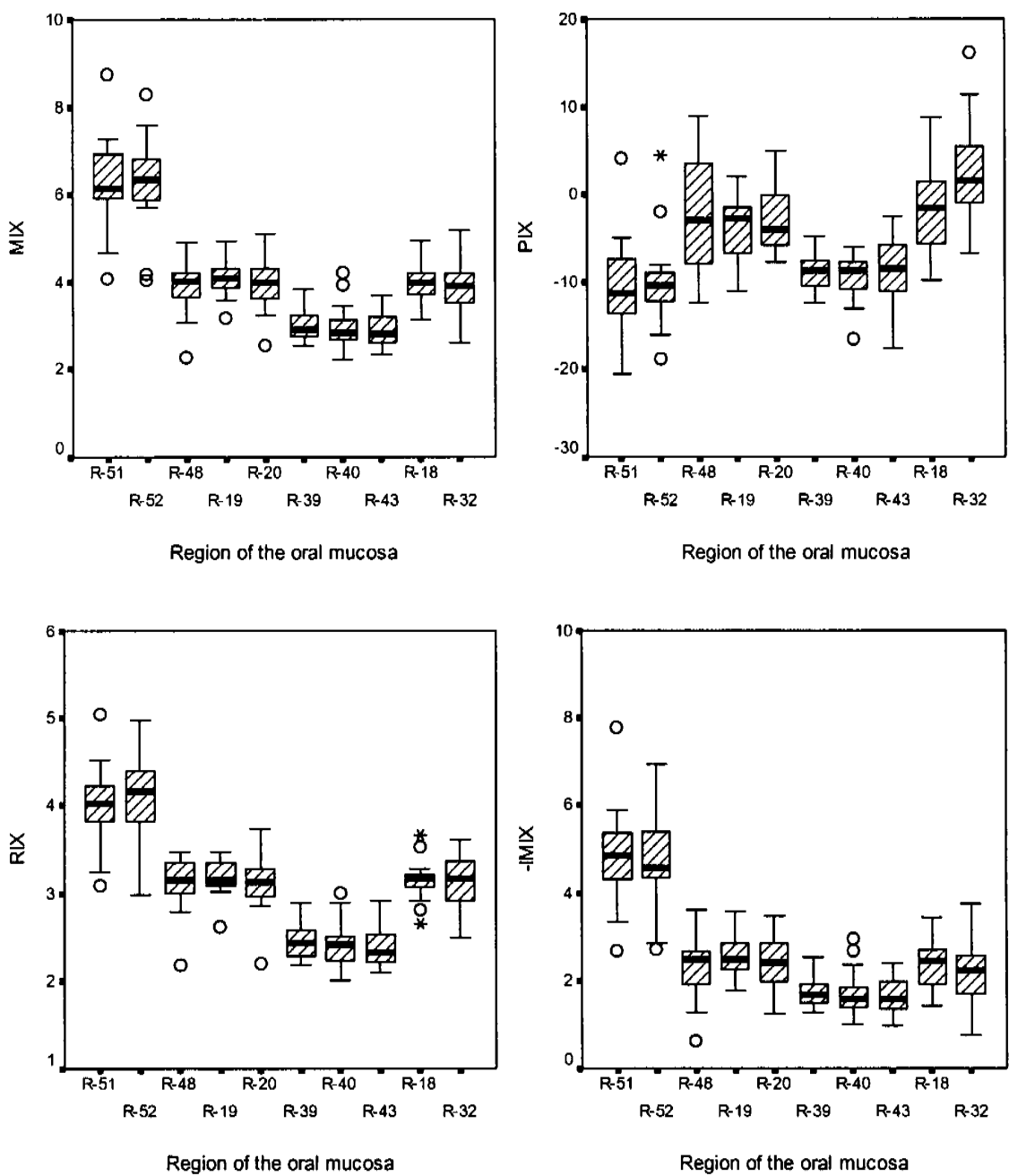

Figure 3: Impedance indices $M I X, P I X, R L X$ and $I M I X$ at various regions of the oral mucosa. Since $I M I X$ is always less than zero, $-I M I X$ is plotted on the ordinate. The box length represents the interquartile range. A line across the box indicates the median. The whiskers extend to the highest and lowest values, excluding outliers. Circles represent outliers (cases with values between 1.5 and 3 box lengths from the upper or lower edge of the box) and asterisks represent extremes (cases with more than 3 box lengths from the upper or lower edge of the box). 


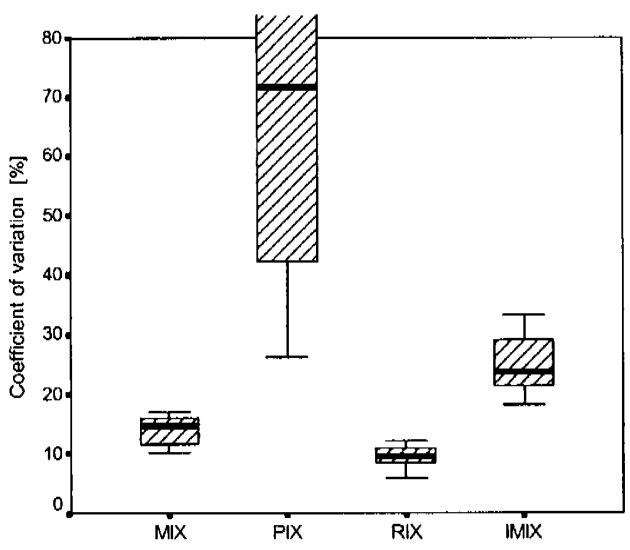

Figure 4: Coefficient of variation of the impedance indices over studied regions of the oral mucosa. The plot is broken intentionally since variation of PIX spans up to $390 \%$. Symbols mean the same as in Figure 3.

The coefficients of variation for impedance indices summarized for all studied regions are shown in Figure 4. The plot is broken intentionally since variation of $P I X$ spans up to $390 \%$. Such a high variation means that no reference values for PIX could be established and that its applicability is strictly limited to cases when reference from the same subject is used. Reference values for other indices could be established, but their variation is far from being unimportant. These results show that the applicability of the impedance indices, when no reference from the same subject is used, is much more limited than was believed [10].

\section{Conclusions}

Impedance measurements in the oral cavity were not performed until recently. The main obstacles were the design of impedance probe for intraoral measurement and wet environment of the oral cavity. As with all impedance measurements in vivo it is beneficial that everyone is taken as its own reference, but for many real applications that is not possible. Specific for our study is that the experimental group was composed of mostly middle-aged and elderly subjects what is favourable, since the oral disorders are most prevalent in this population. The main conclusions of our study can be summarized as follows: the proposed set of indices is not fully satisfactory nor could be generally accepted as data reduction technique; applicability of these impedance indices seems limited mainly to cases when everyone is taken as its own reference. Otherwise only $M I X$ can be recommended. Moreover, it appears that indices' behaviour depend not only on the properties of tissue, but also on the characteristics of impedance probe being used. Our future work will include estimation of probe influence on measurement, optimisation of indices and collection of impedance data from patients with oral disorders. 